\title{
Prospective relationship between objectively measured light physical activity and depressive
}

\section{symptoms in later life}

Running title: Objectively measured light physical activity and depressive symptoms

Submission to International Journal of Geriatric Psychiatry

1st author: Po-Wen $\mathrm{Ku}, \mathrm{PhD}$ (Epidemiology), $\mathrm{PhD}$ (Exercise \& Health)

Graduate Institute of Sports and Health, National Changhua University of Education, Taiwan Department of Epidemiology and Public Health, University College London, UK

Email: powen.ku@gmail.com

2nd Author: Andrew Steptoe, DPhil, DSc

Department of Epidemiology and Public Health, University College London, UK

Email: a.steptoe@ucl.ac.uk

3rd Author: Yung Liao, PhD

Department of Health Promotion and Health Education, National Taiwan Normal University, Taiwan

E-mail: liaoyung@ntnu.edu.tw

4th Author: Wen-Jung Sun, MD, PhD

Family Medicine Department, Taipei City Hospital Zhongxing Branch, Taiwan

E-mail: das48@tpech.gov.tw

5th and Corresponding Author: Li-Jung Chen, $\mathrm{PhD}$

Department of Exercise Health Science, National Taiwan University of Sport, Taiwan

Department of Epidemiology and Public Health, University College London, UK

No. 16, Section 1, Shuang-Shih Rd., Taichung, 404 Taiwan

E-mail: 1jchen@ntupes.edu.tw; Telephone: +886 (4) 2221-3135 ext.1310

Word count: 2,734 (without including tables and references); abstract: 245

Number of tables: 4

The authors declare no conflicts of interest 


\title{
Prospective relationship between objectively measured light physical activity and depressive
}

\section{symptoms in older adults}

\begin{abstract}
Background: The use of self-report measures of physical activity is a serious methodological weakness in many studies of physical activity and depressive symptoms. It is still equivocal whether light physical activity protects older adults from depressive symptoms.
\end{abstract}

Objective: This study aimed to explore whether objectively measured light physical activity, independent of sedentary and moderate-to-vigorous activity, is associated with a reduced risk of subsequent depressive symptoms in older adults.

Methods: This was a two-year prospective cohort study. A total of 285 community-dwelling older adults aged 65 or older were interviewed in 2012. A second wave of assessment was carried out in 2014 involving 274 (96.1\%) participants. Time spent in physical activity at different intensities was assessed using tri-axial accelerometers. Depressive symptoms were measured using the 15-item Geriatric Depression Scale. Negative binomial regression models with adjustment for baseline depressive symptoms, accelerometer wear time, socio-demographic variables, lifestyle behaviors, and chronic disease conditions, were conducted.

Results: Time spent in moderate-to-vigorous and light physical activities were both inversely related to depressive symptoms at follow-up. Sedentary time was associated with an increased 
risk of subsequent depressive symptoms. When sedentary or moderate-to-vigorous activity were included in the multivariable adjusted regression models with light physical activity simultaneously, only light physical activity remained significant. Sensitivity analyses for assessing confounding and reverse causation provided further support for the stability of these findings.

Conclusion: Light physical activity, independent of sedentary and moderate-to-vigorous activity, is associated with a reduced risk of subsequent depressive symptoms in later life.

Key words: Exercise, Depression, Longitudinal, Accelerometer, Objective measure 


\section{Introduction}

Depression is a common mental problem, characterized by sadness, diminished interest or pleasure, fatigue or loss of energy, disturbed sleep or appetite, feelings of worthlessness or inappropriate guilt, and poor concentration (American Psychiatric Association 2013). It is a leading cause of disability worldwide and is an important contributor to the overall global burden of disease (Ferrari, et al. 2013). A systematic review of depression in later life estimated a pooled prevalence for major depression of $7.2 \%$ and $17.1 \%$ for depressive symptoms (Luppa, et al. 2012). Although depression is less prevalent in older adults than in younger adults, it may lead to serious consequences such as increased risk of morbidity and mortality, decreased physical, cognitive and social functioning, and greater self-neglect (Blazer 2003; Fiske, et al. 2009). This underscores the need to identify correlates or determinants such as modifiable lifestyle behaviors that can prevent or delay the onset of depressive disorders among aging populations.

Systematic reviews and meta-analyses of prospective studies suggest that there is an inverse relationship between physical activity and depression/depressive symptoms in older adults (Mammen and Faulkner 2013). However, the use of self-report measures of physical activity is a serious methodological weakness in many studies. Although self-report instruments are easy to administer and are inexpensive, they may be subject to recall bias. Self-report questionnaires may be not sensitive to age, culture, and ethnicity. Additionally, these measures tend to have acceptable reliability $(>0.7)$ but only low to moderate validity as assessed against accelerometer 
assessments (Murphy 2009).

International physical activity guidelines recommend that older adults should do at least 150 minutes of moderate-intensity aerobic physical activity throughout the week or do at least 75 minutes of vigorous intensity aerobic physical activity throughout the week or an equivalent combination of moderate- and vigorous-intensity activity (World Health Organization 2010). This appears to imply that little or no health benefits can be derived from light physical activities (e.g. walking slowly, lifting lightweight objects, household chores, yoga, and Tai Chi etc.). However, these recommendations are mainly based on large-scale epidemiologic studies of self-reported physical activity in relation to all-cause mortality and cardiometabolic factors such as cardiovascular disease, type 2 diabetes, and measures of blood pressure, lipid, glucose and insulin (Lee and Shiroma 2014). They are not based on mental health outcomes. One cross-sectional study has demonstrated that objectively assessed light physical activity is associated with a reduced risk of depressive symptoms among older adults, but it did not adjust statistically for moderate-to-vigorous physical activities (Loprinzi 2013). To date, there have been no longitudinal studies examining whether objectively measured light physical activity, independent of moderate-to-vigorous physical activity, is prospectively related to a decreased risk of subsequent depressive symptoms at older ages.

To fill this gap in literature, our study explored whether objectively assessed light physical activity, independent of other intensities of physical activity, was prospectively associated with 
depressive symptoms among community-dwelling older adults in Taiwan over a 2-year follow-up period. We also included potentially confounding factors in multivariable analyses, and conducted sensitivity analyses to test for confounding and reverse causation.

\section{Methods}

\section{Study design and sample}

A total of 285 community-dwelling older adults aged 65 or older (mean age $=74.5$ years, $\mathrm{SD}=6.1$ years) were recruited and assessed from August to October 2012 in Hunei District, Kaohsiung, which is the second largest city in Taiwan. Of the 14 villages in the district, approximate 20 participants were recruited from each community center at each village using quota sampling (national distribution of population according to sex and age in 2011) (Taiwan Ministry of Interior 2012). The data were collected through standardized household face-to-face interviews and accelerometry. Of the initial sample, 274 (96.1\%) attended the second-wave interviews from May to July 2014 (mean follow-up time $=22.1 \pm 1.5$ months). All participants provided written consent at the time of enrollment. Ethical approval for this study was provided from National Taiwan University of Sport Institutional Review Board, Taiwan.

\section{Measures}

\section{Depressive symptoms}

The 15-item Geriatric Depression Scale (GDS) was utilized to assess depressive symptoms 
with a potential range of 0 to 15 (Brink, et al. 1982; Yesavage and Sheikh 1986). The Chinese version of this scale has demonstrated adequate reliability and validity among community-dwelling older adults in Taiwan (Liu, et al. 1997; Wong, et al. 2002). The Cronbach's alpha reliability coefficients for the 15-item GDS ranged between 0.74 and 0.84 in the two waves of data collection.

Time spent in physical activity at different intensities

Time spent in physical activity at different intensities was objectively measured using waist worn triaxial accelerometer monitors (GT3X+, ActiGraph, Pensacola, FL, USA) for 7 days. Participants were required to wear the sensor at least 10 hours of monitoring on at least 5 days (Buman, et al. 2010). Data were analyzed and scored using the ActiLife 6.2 software. Periods of $60 \mathrm{~min}$ of consecutive zero counts were considered as non-wearing time and were excluded from the analyses. Several physical activity parameters were then created, including time spent in sedentary behaviors ( $<100$ counts/min), light physical activity (100-1951 counts/min), and moderate-to-vigorous activity (>1951 counts/min) (Gorman, et al. 2014), together with total physical activity energy expenditure (kcal/week). For descriptive purposes, the various physical activity categories were each divided into tertiles, but the primary analyses were conducted on continuously distributed variables.

\section{Covariates}

The following factors in the 2012 baseline survey were incorporated as covariates according 
to previous work (Mammen and Faulkner 2013): (i) socio-demographic factors: sex, age $(<75$, 75+), educational attainment (no formal schooling, primary school, secondary school+), marital status (married/cohabitating, others), main source of income (from offspring vs. self [e.g. pension/savings]); (ii) lifestyle behaviors: smoker (current, never, or former smokers), alcohol consumption (yes vs. no), and; (iii) health status: body mass index (BMI) $(<18.50,18.50-23.99$, 24-26.99, 27+) (Taiwan Department of Health 2003), number of chronic diseases $(0,1,2+)$ :

Participants were presented with a list of chronic conditions (i.e. hypertension, stroke, diabetes, heart disease, cancer, chronic obstructive pulmonary disease (COPD), liver disease, renal disease, and arthritis) and were asked to indicate whether they have been diagnosed with each condition by a clinician. The total number of self-reported chronic conditions were then computed; difficulties with activities of daily living (ADLs, no difficulties at all vs. some or great difficulties); insomnia measured by the Athens Insomnia Scale (no vs. yes) (Chiang, et al. 2009); cognitive impairment assessed using the mini mental status examination (MMSE). Cut-off points of cognitive impairment were the scores of the MMSE $<14$ for those with no formal schooling, and MMSE $<24$ for those with primary school level or higher (Guo, et al. 1988); mean daily accelerometer wear time (Hamer, et al. 2014), and baseline depressive symptoms scores.

\section{Data analysis}

Descriptive statistics were used to describe the features of the study sample. Given the violation of normality, Mann Whitney U tests and Kruskal-Wallis tests were adopted to check for 
differences in depressive symptom scores in 2014 across levels of accelerometer-derived parameters (all in tertiles), and covariates. Variables with a $p$ value less than 0.25 were included in the subsequent regression models for adjustment (Hosmer, et al. 2013).

To examine the crude correlations between objectively assessed physical activity parameters and subsequent depressive symptoms after controlling for accelerometer wear time, partial Spearman correlation coefficients between physical activity energy expenditure ( $\mathrm{kcal} / \mathrm{week}$ as a continuous variable), time (hours/day as a continuous variable) spent in physical activity at different intensities, and subsequent depressive symptoms were computed.

To assess the adjusted associations between physical activity parameters and subsequent depressive symptoms, multivariable negative binomial regression was conducted because the outcome variable was an over-dispersed count with a highly skewed distribution. All accelerometer-derived parameters were log-transformed before conducting regression analyses due to non-normality (Tudor-Locke, et al. 2011). Three separate wear time-adjusted regression models (single-factor models) for sedentary, light, and moderate-to-vigorous activity were conducted to assess the associations between each intensity categories and depressive symptoms. Then, two wear time-adjusted regression models (two-factor models) for moderate-to-vigorous and light activity, and sedentary and light activity respectively were fitted to examine the relationships in more detail. To avoid multi-collinearity, time spent in moderate-to-vigorous, light, and sedentary behaviors were not entered into the models at the same time because accelerometer 
wear time is equal to the sum of these behaviors.

Sensitivity analyses were carried out to evaluate confounding and reverse causation. Firstly, we considered the possibility that ADL difficulties might influence physical activity behaviors at baseline and subsequent depressive symptoms, so the negative binomial regressions were repeated after excluding the 13 participants with impaired ADLs. Secondly, we mitigated the potential impact of prodromal cognitive deterioration on physical activity and depressive symptoms at baseline by excluding participants with cognitive impairment $(n=34)$ at baseline (Guo et al. 1988).

Multicollinearity was checked using multiple linear regression (i.e., variance inflation factor value $>5$ or tolerance value $<0.2$ ) (Belsley, et al. 1980; de Vaus 2002), and the results indicate that this was not a problem in these regression models. All analyses were conducted using IBM SPSS 20.0 software and a $p$ value $<0.05$ was considered statistically significant.

\section{Results}

\section{Characteristics of the sample}

The differences in depressive symptoms at follow-up categorized by baseline characteristics are presented in Table 1. Individuals who had lower levels of depressive symptoms in 2014 were more likely to male, alcohol drinkers, and have mild or no comorbidities, cognitive impairment, or depressive symptoms at baseline $(p<0.05)$. Overall, participants spent $8.72 \pm(\mathrm{SD}) 3.08$ 
hour/day in sedentary activities, $3.75 \pm 1.74$ hour/day in light intensity activity, and $0.16 \pm 0.62$ hour/day in moderate-to-vigorous intensity physical activity. Participants with fewer depressive symptoms in 2014 had higher levels of total physical activity energy expenditure and spent more time in moderate-to-vigorous and light physical activity than those who were less active in 2012.

Table 1 here

\section{Spearman's correlations between physical activity and subsequent depressive symptoms}

Physical activity energy expenditure $(\rho=-0.38)$ and time spent in moderate-to-vigorous $(\rho=$ $-0.24)$, and light physical activities $(\rho=-0.30)$ were all inversely associated with subsequent depressive symptoms, whereas sedentary time $(\rho=0.27)$ was positively related to depressive symptoms $(p<0.001)$. Overall, the magnitude of associations between physical activity parameters and depressive symptoms were small-to-moderate.

Multivariable adjusted associations between physical activity and subsequent depressive symptoms

The multivariable adjusted regression (single-factor models) showed that participants who 
spent more time in physical activity at moderate-to-vigorous (Rate Ratio $[\mathrm{RR}]=0.88,95 \% \mathrm{CI}=$ $0.79-0.98)$ or light intensities $(\mathrm{RR}=0.63,95 \% \mathrm{CI}=0.49-0.82)$ had fewer depressive symptoms at follow-up. In contrast, individuals engaging in more sedentary behaviors had higher levels of subsequent depressive symptoms $(\mathrm{RR}=2.45,95 \% \mathrm{CI}=1.16-5.15) .($ See Table 2$)$

Table 2 here

When moderate-to-vigorous physical activity and light physical activity were both included in the two-factor model, only light physical activity remained significant. Similarly, light physical activity was a stronger predictor of subsequent depressive symptoms than sedentary time in the second two-factor model. (See Table 3)

Table 3 here 


\section{Sensitivity analysis}

The sensitivity analyses are detailed in Table 4. The association patterns for the single-factor models or the two-factor models were similar to those in Table 3 after excluding participants with ADL difficulties. Although the sample size quite markedly reduced after further excluding those with cognitive impairment, the patterns of associations did not alter very much. In each stage, greater light physical activity was associated with fewer depressive symptoms at follow-up.

Table 4 here

\section{Discussion}

This two-year study revealed that time spent in moderate-to-vigorous and light physical activities were both inversely related to depressive symptoms. In contrast, more sedentary time was associated with higher levels of subsequent depressive symptoms. Additionally, when sedentary or moderate-to-vigorous activity was entered into the multivariable adjusted regression models with light activity simultaneously, only light physical activity remained significant. These prospective results were verified even when adjusting for underlying covariates, including 
baseline depressive symptoms, chronic disease conditions, and accelerometer wear time.

Sensitivity analyses for scrutinizing confounding and reverse causation provided further evidence for the stability of these findings.

Although self-report physical activity instruments are cost-effective, they are more reliable and valid for assessing moderate-to-vigorous intensity instead of light-intensity activities. Epidemiological studies based on self-reports have tended to focus on their measures of moderate-to-vigorous activities (Lee and Shiroma 2014). Unfortunately, the use of different self-report instruments may yield conflicting results (Teychenne, et al. 2008) and restrict the capacity to assess the relations between physical activity at different intensities and depressive symptoms (Mammen and Faulkner 2013).

There is currently no evidence on the longitudinal relationship between objectively measured light physical activity and depressive symptoms in older adults. The present study indicated that the associations were stronger for light activity than for moderate-to-vigorous activity. This supports earlier prospective work suggesting that light-intensity physical activity is a stronger predictor of mental well-being than moderate-to-vigorous physical activity in older adults (Buman et al. 2010). Compared with light activity, relatively fewer older people engage in moderate/vigorous activity. This might in part explain the weaker associations between moderate/vigorous activity and depressive symptoms. An alternative explanation is that light physical activity may be more beneficial for mental health because more discomfort and short of 
breath may be generated while engaging in moderate/vigorous physical activity (Hamer et al. 2014), especially in older people.

This is an observational study, so cannot establish causality. However, the beneficial effect of light physical activity on mental health in older populations has been observed in intervention studies as well. Several studies have been carried out, with the greatest improvements in mood being associated with light intensity exercise among older adults (Arent, et al. 2000). Another systematic review of randomized controlled trials in older adults suggested that two sessions per week of 'light to moderate' intensity each of a minimum of 45 min duration were more appropriate for improving older adults' subjective well-being than more intense moderate-to-vigorous activity (Windle, et al. 2010). Given the problems of motivation and adherence to moderate-to-vigorous activity in older populations (Sparling, et al. 2015), light physical activity may be more feasible and help people achieve mental health benefits.

Sedentary behaviors are prevalent among older populations, and are increasingly regarded as a serious threat to healthy aging (de Rezende, et al. 2014). Our study showed that sedentary time is related to an increased risk of depressive symptoms, which is consistent with the findings from previous work (Teychenne, et al. 2010). We further assessed the independent associations of sedentary and light activity with depressive symptoms. The results revealed that light activity is a stronger correlate of depressive symptoms than sedentary behaviors. This highlights the importance of reducing sedentary behaviors by introducing light activity throughout the day for 
improving older adults' mental health.

To our knowledge, this is the first study documenting the prospective associations between objectively assessed light physical activity and depressive symptoms in older adults. Although this research was conducted adjusting for multiple potential confounders and included sensitivity analyses to address other influences on outcomes, it inevitably has some limitations. First, this study was based on community-dwelling older adults using a sex-age quota sampling and an excellent response rate on follow-up, but there may still be problems of sample representativeness. Compared with a previous study using the Taiwan National Health Interview Survey, we found there were no significant differences in terms of education level, but that participants in the current study had less cognitive impairment (Ku, et al. 2012), less difficulty in ADL and fewer chronic conditions (data not shown) (Chen, et al. 2012). Because inactivity and depressive symptoms may be less prevalent in this healthier subgroup of the population, potential selection bias may have led to underestimation of the association between physical activity and depressive symptoms. A well-designed longitudinal study using a nationally representative sample is warranted to verify this association and compare the effects of light physical activity on subsequent depressive symptoms among those with physical limitations, cognitive impairment, and multiple chronic conditions. Furthermore, this research cannot establish causality given that it is an observational study. The average amount of moderate-to-vigorous physical activity was small, and restricted variability may have limited the scope for identifying associations with 
depressive symptoms.

In sum, this study expands our understanding of how time spent in physical activity at different intensities is related to depressive symptoms in older adults. It presents evidence that light physical activity, independent of sedentary and moderate-to-vigorous activity, is associated with a reduced risk of subsequent depressive symptoms. This extends the existing evidence for the benefits of physical activity for disease prevention and improvement of mental well-being. 


\section{Acknowledgements}

The authors declare no conflicts of interest and acknowledge funding support from Taiwan

Ministry of Science and Technology (MOST 105-2628-H-018-001-MY2)

Conflict of interest: The authors do not have any conflict of interest to report.

Ethics statement: Ethics approval for this study was obtained from National Taiwan University of Sport Institutional Review Board, Taiwan (NTUPES-HSC-100-09). 


\section{References}

American Psychiatric Association 2013 Diagnostic and statistical manual of mental disorders. Washington, DC: American Psychiatric Association.

Arent SM, Landers DM \& Etnier JL 2000 The effects of exercise on mood in older adults: a meta-analytic review. J Aging Phys Act 8: 407-430.

Belsley DA, Kuh E \& Welsch RE 1980 Regression diagnostics: identifying influential data and sources of collinearity. New York: John Wiley \& Sons.

Blazer DG 2003 Depression in late life: review and commentary. J Gerontol A Biol Sci Med Sci 58: M249-M265.

Brink TL, Yesavage JA, Lum O, Heersema PH, Adey M \& Rose TL 1982 Screening tests for geriatric depression. Clin. Gerontol. 1: 37-43.

Buman M, Hekler E, Haskell W, Pruitt L, Conway T, Cain K, Sallis J, Saelens B, Frank L \& King A 2010 Objective light-intensity physical activity associations with rated health in older adults. Am J Epidemiol 172: 1155-1165.

Chen LJ, Stevinson C, Ku PW, Chang YK \& Chu DC 2012 Relationships of leisure-time and non-leisure-time physical activity with depressive symptoms: a population-based study of Taiwanese older adults. Int J Behav Nutr Phys Act 9: 1-10.

Chiang HL, Chen HC, Bai CHC, H H, Lee MB, Lai SH \& Chou P 2009 A validation study of the Chinese version of the Athens insomnia scale. Taiwan J of Psychiatry 23: 43-52.

de Rezende LF, Rey-Lopez J, Matsudo VK \& Luiz O 2014 Sedentary behavior and health outcomes among older adults: a systematic review. BMC Public Health 14: 333.

de Vaus D 2002 Analyzing social science data: 50 key problems in data analysis. London: Sage. Ferrari AJ, Charlson FJ, Norman RE, Patten SB, Freedman G, Murray CJL, Vos T \& Whiteford HA 2013 Burden of depressive disorders by country, sex, age, and year: Findings from the Global Burden of Disease Study 2010. PLoS Med 10: e1001547. 
Fiske A, Wetherell JL \& Gatz M 2009 Depression in older adults. Ann Rev Clin Psych 5: 363-389.

Gorman E, Hanson H, Yang P, Khan K, Liu-Ambrose T \& Ashe M 2014 Accelerometry analysis of physical activity and sedentary behavior in older adults: A systematic review and data analysis. Eur Rev Aging Phys Act 11: 35-49.

Guo NW, Liu HC, Wong PF, Liao KK, Yan SH, Lin KP, Chang CY \& Hsu TC 1988 Chinese Version and Norms of the Mini-Mental State Examination. J of Rehabil Med Assoc 52-59.

Hamer M, Coombs N \& Stamatakis E 2014 Associations between objectively assessed and self-reported sedentary time with mental health in adults: an analysis of data from the Health Survey for England. BMJ Open 4.

Hosmer DW, Lemeshow S \& Sturdivant RX 2013 Applied logistic regression. NJ: John Wiley \& Sons.

Ku PW, Fox KR, Chen LJ \& Chou P 2012 Associations of leisure, work-related and domestic physical activity with cognitive impairment in older adults. Int J Sports Psychol 43: 103-116.

Lee I-M \& Shiroma EJ 2014 Using accelerometers to measure physical activity in large-scale epidemiological studies: Issues and challenges. Br J of Sports Med 48: 197-201.

Liu C, Wang S, Teng E, Fuh J, Lin C, Lin K, Chen H, Lin C, Wang P \& Yang Y 1997 Depressive disorders among older residents in a Chinese rural community. Psychol Med 27: 943-949.

Loprinzi PD 2013 Objectively measured light and moderate-to-vigorous physical activity is associated with lower depression levels among older US adults. Aging ment health 17: 801-805.

Luppa M, Sikorski C, Luck T, Ehreke L, Konnopka A, Wiese B, Weyerer S, König HH \& Riedel-Heller SG 2012 Age- and gender-specific prevalence of depression in latest-life Systematic review and meta-analysis. J of Affect Disord 136: 212-221.

Mammen G \& Faulkner G 2013 Physical activity and the prevention of depression: A systematic review of prospective studies. Am J Prev Med 45: 649-657.

Murphy SL 2009 Review of physical activity measurement using accelerometers in older adults: 
considerations for research design and conduct. Prev Med 48: 108-114.

Sparling PB, Howard BJ, Dunstan DW \& Owen N 2015 Recommendations for physical activity in older adults. BMJ 350: h100.

Taiwan Department of Health 2003 Identification, Evaluation, and Treatment of Overweight and Obesity in Adults in Taiwan. Taipei: Taiwan Department of Health.

Taiwan Ministry of Interior 2012 Monthly Bulletin of Interior Statistics: Resident Population by Age Group. Taipei: Taiwan Ministry of Interior.

Teychenne M, Ball K \& Salmo J 2010 Sedentary Behavior and Depression Among Adults: A Review. Int J Behav Med 17: 246-254.

Teychenne M, Ball K \& Salmon J 2008 Physical activity and likelihood of depression in adults: A review. Prev Med 46: 397-411.

Tudor-Locke C, Leonardi C, Johnson WD, Katzmarzyk PT \& Church TS 2011 Accelerometer steps/day translation of moderate-to-vigorous activity. Prev Med 53: 31-33.

Windle G, Hughes D, Linck P, Russell I \& Woods B 2010 Is exercise effective in promoting mental well-being in older age? A systematic review. Aging Ment Health 14: 652-669.

Wong M, Ho T, Ho M, Yu C, Wong Y \& Lee S 2002 Development and inter rater reliability of a standardized verbal instruction manual for the Chinese Geriatric Depression Scale short form. Int J of Geriatr Psychiatry 17: 459-463.

World Health Organization 2010 Global recommendations on physical activity for health. Geneva: World Health Organization.

Yesavage JA \& Sheikh JI 1986 Geriatric Depression Scale (GDS): Recent evidence and development of a shorter version. In Clinical gerontologist: A Guide to Assessment and Intervention, pp 165-173. Ed TL Brink. New York: The Haworth Press. 
Table 1 Characteristics of participants in 2012 with depressive symptoms in 2014

\begin{tabular}{|c|c|c|c|}
\hline Variables in 2012 & $\mathrm{n}(\%)$ & $\begin{array}{l}\text { Depressive Symptoms } \\
\text { in } 2014(\text { Mean } \pm \text { SD) }\end{array}$ & $p$-value ${ }^{\mathrm{a}}$ \\
\hline \multicolumn{4}{|l|}{ Socio-demographic } \\
\hline Sex & & & 0.014 \\
\hline Male & $125(45.6)$ & $2.02(2.01)$ & \\
\hline Female & $149(54.4)$ & $2.83(2.91)$ & \\
\hline Age & & & 0.085 \\
\hline$<75$ & $156(56.9)$ & $2.29(2.39)$ & \\
\hline $75+$ & $118(43.1)$ & $2.69(2.78)$ & \\
\hline Education level & & & 0.353 \\
\hline Secondary school+ & $48(17.6)$ & $1.79(1.32)$ & \\
\hline Primary school & $113(41.2)$ & $2.45(2.45)$ & \\
\hline No formal schooling & $113(41.2)$ & $2.76(3.01)$ & \\
\hline Marital status & & & 0.645 \\
\hline Married & $192(70.1)$ & $2.33(2.28)$ & \\
\hline Others & $82(29.9)$ & $2.77(3.12)$ & \\
\hline Main source of income & & & 0.187 \\
\hline Offspring & $139(50.7)$ & $2.23(2.24)$ & \\
\hline Self (pension/savings) & $135(49.3)$ & $2.70(2.85)$ & \\
\hline \multicolumn{4}{|l|}{ Lifestyle behaviors } \\
\hline Total energy expenditures (kcal/week) & & & $<0.001$ \\
\hline High & $92(33.6)$ & $1.61(1.61)$ & \\
\hline Medium & $91(33.2)$ & $2.25(2.36)$ & \\
\hline Low & $91(33.2)$ & $3.54(3.13)$ & \\
\hline Moderate-to-vigorous PA (hour/day) & & & $<0.001$ \\
\hline High & $92(33.6)$ & $1.76(1.82)$ & \\
\hline Medium & $88(32.1)$ & $2.26(2.14)$ & \\
\hline Low & $94(34.3)$ & $3.34(3.24)$ & \\
\hline Light PA (hour/day) & & & $<0.001$ \\
\hline High & $93(33.9)$ & $2.04(2.47)$ & \\
\hline Medium & $91(33.2)$ & $2.16(2.15)$ & \\
\hline Low & $90(32.9)$ & $3.20(2.90)$ & \\
\hline Sedentary time (hour/day) & & & 0.528 \\
\hline
\end{tabular}


Low

Medium

High

Smoking

Current smoker

Never smoker

Former smoker

Drinking

Yes

No

Health Status

Body mass index

$\begin{array}{lc}\text { Underweight } & <18.5 \\ \text { Normal } & 18.5-23.99 \\ \text { Overweight } & 24-26.99 \\ \text { Obese } & 27+\end{array}$

Number of chronic diseases

0

1

$2+$

Insomnia

No

Yes

Activities of daily living

No difficulty at all

Some or great

Cognitive impairment

No

Yes
89 (32.5)

92 (33.6)

93 (33.9)

$22(8.0)$

238 (86.9)

$14(5.1)$

13 (4.7)

261 (95.3)
2.54 (2.76)

2.33 (2.52)

$2.53(2.45)$

0.576

$2.00(1.60)$

2.49 (2.66)

2.79 (2.26)

0.012

1.31 (1.49)

$2.52(2.60)$

0.596

$\begin{array}{rr}11(4.0) & 2.55(2.34) \\ 101(36.9) & 2.58(2.41) \\ 101(36.9) & 2.45(2.84) \\ 61(22.2) & 2.28(2.44)\end{array}$

$118(43.0)$

0.019

104 (38.0)

$1.94(1.83)$

52 (19.0)

2.79 (2.66)

3.00 (2.49)

0.058

225 (82.1)

2.28 (2.36)

49 (17.9)

3.33 (3.24)

0.003

$261(92.3)$

2.37 (2.51)

$13(7.7)$

4.31 (3.04)

0.001

240 (87.6)

$2.30(2.44)$

34 (12.4)

3.65 (3.14)

274 (100.0)

$\rho=0.19^{b}$

$0.001^{\mathrm{b}}$

a: Mann Whitney U test or Kruskal-Wallis test

b: Spearman's correlation 
Table 2 Multivariable adjusted regression of the single-factor models examining the independent effects of time spent in different intensities of physical activity at baseline on subsequent depressive symptoms $(n=274)$

\begin{tabular}{|c|c|c|c|c|c|c|}
\hline Variables & $\mathrm{RR}(95 \% \mathrm{CI})$ & $p$ & $\mathrm{RR}(95 \% \mathrm{CI})$ & $p$ & $\mathrm{RR}(95 \% \mathrm{CI})$ & $p$ \\
\hline \multicolumn{7}{|l|}{ Physical activity } \\
\hline MVPA & $0.88(0.79-0.98)$ & 0.021 & & & & \\
\hline Sedentary & & & & & $2.45(1.16-5.15)$ & 0.019 \\
\hline \multicolumn{7}{|l|}{ Covariates } \\
\hline Sex (male vs. female) & $0.89(0.65-1.21)$ & 0.45 & $0.78(0.56-1.06)$ & 0.12 & $0.80(0.58-1.10)$ & 0.17 \\
\hline Drinking (yes vs. no) & $0.61(0.28-1.35)$ & 0.23 & $0.60(0.27-1.33)$ & 0.21 & $0.58(0.26-1.29)$ & 0.19 \\
\hline \multicolumn{7}{|l|}{ Number of diseases } \\
\hline 0 (vs. $2+)$ & $0.72(0.48-1.09)$ & 0.12 & $0.89(0.58-1.35)$ & 0.57 & $0.80(0.53-1.20)$ & 0.28 \\
\hline
\end{tabular}




\begin{tabular}{lcccccc}
1 (vs. 2+) & $0.93(0.61-1.40)$ & 0.71 & $1.06(0.70-1.60)$ & 0.78 & $0.97(0.65-1.47)$ & 0.90 \\
Insomnia (no vs. yes) & $0.72(0.49-1.05)$ & 0.09 & $0.69(0.47-1.02)$ & 0.07 & $0.71(0.48-1.05)$ & 0.09 \\
ADL difficulty (no vs. yes) & $0.58(0.29-1.17)$ & 0.13 & $0.64(0.32-1.27)$ & 0.20 & $0.63(0.32-1.26)$ & 0.19 \\
Cognitive impairment (no vs. yes) & $0.83(0.53-1.29)$ & 0.40 & $0.85(0.54-1.33)$ & 0.48 & $0.81(0.52-1.26)$ & 0.35 \\
Accelerometer wearing time & $0.96(0.91-1.01)$ & 0.15 & $0.98(0.93-1.04)$ & 0.46 & $0.88(0.81-0.95)$ & 0.002 \\
Baseline depressive symptoms & $1.07(1.00-1.14)$ & 0.04 & $1.07(1.00-1.13)$ & 0.04 & $1.08(1.01-1.14)$ & 0.02 \\
\hline
\end{tabular}

$\mathrm{RR}=$ rate ratio; $\mathrm{MVPA}=$ moderate-to-vigorous physical activity; $\mathrm{LPA}=$ light physical activity, $\mathrm{ADL}=$ activities of daily living

a: The three single-factor models represent the association of each intensity category (MVPA, LPA, and sedentary time) with depressive symptoms without mutual adjustment for other categories of activity 
Table 3 Multivariable adjusted regression of the two-factor models examining the independent effects of time spent in different intensities of physical activity at baseline on subsequent depressive symptoms $(n=274)$

\begin{tabular}{lcccc}
\hline \multirow{2}{*}{\begin{tabular}{l} 
Variables \\
\cline { 2 - 4 }
\end{tabular}} & \multicolumn{4}{l}{ Two-factor models $^{\mathrm{a}}$} \\
\hline Physical activity $(95 \% \mathrm{CI})$ & $p$ & $\mathrm{RR}(95 \% \mathrm{CI})$ & $p$ \\
MVPA & $0.94(0.84-1.05)$ & 0.288 & & \\
LPA & $0.67(0.51-0.88)$ & 0.004 & $0.66(0.48-0.91)$ & 0.011 \\
Sedentary & & $1.20(0.47-3.08)$ & 0.703 \\
\hline
\end{tabular}

$\mathrm{RR}=$ rate ratio; $\mathrm{MVPA}=$ moderate-to-vigorous physical activity $\mathrm{LPA}=$ light physical activity

Covariates in two models: baseline depressive symptoms, sex, age, income, alcohol consumption, number of chronic diseases, activities of daily living, insomnia, and cognitive impairment

a: The two-factor models further adjusted for MPVA (or sedentary time) for LPA. 
Table 4 Sensitivity analyses of the single-factor and two-factor regression models examining the independent effects of time spent in different intensities of physical activity at baseline on subsequent depressive symptoms

Stage 1: Excluding participants with ADL difficulties at baseline $(n=261)$

\begin{tabular}{|c|c|c|c|c|c|c|}
\hline \multirow{2}{*}{ Physical activity } & \multicolumn{2}{|c|}{ Single-factor models ${ }^{\mathrm{a}}$} & \multicolumn{4}{|c|}{ Two-factor models ${ }^{b}$} \\
\hline & $\mathrm{RR}(95 \% \mathrm{CI})$ & $p$ & $\mathrm{RR}(95 \% \mathrm{CI})$ & $p$ & $\mathrm{RR}(95 \% \mathrm{CI})$ & $p$ \\
\hline MVPA & $0.88(0.78-0.98)$ & 0.018 & $0.93(0.83-1.05)$ & 0.239 & & \\
\hline LPA & $0.62(0.47-0.82)$ & $<0.001$ & $0.66(0.50-0.88)$ & 0.004 & $0.65(0.47-0.91)$ & 0.011 \\
\hline Sedentary & $2.55(1.18-5.49)$ & 0.017 & & & $1.25(0.48-3.27)$ & 0.651 \\
\hline
\end{tabular}

Stage 2: Excluding participants with ADL difficulties and cognitive impairment at baseline $(n=233)$

\begin{tabular}{lllllll}
\hline MVPA & $0.89(0.78-1.00)$ & 0.040 & $0.95(0.83-1.07)$ & 0.375 & & \\
LPA & $0.59(0.43-0.80)$ & $<0.001$ & $0.62(0.44-0.86)$ & 0.004 & $0.61(0.40-0.92)$ & 0.018 \\
Sedentary & $2.84(1.25-6.41)$ & 0.012 & & & $1.15(0.38-3.46)$ & 0.798 \\
\hline
\end{tabular}

$\mathrm{RR}=$ rate ratio; $\mathrm{MVPA}=$ moderate-to-vigorous physical activity; $\mathrm{LPA}=$ light physical activity

Covariates in all models: baseline depressive symptoms, sex, age, income, alcohol consumption, number of chronic diseases, activities of daily living, insomnia, and cognitive impairment

a: The three single-factor models represent the association of each intensity category (MVPA, LPA, and sedentary time) with depressive symptoms without mutual adjustment for other categories of activity

b: The two-factor models further adjusted for MPVA (or sedentary time) for LPA. 